\title{
Educomunicação e comunicação organizacional: um universo de possibilidades e conexões
}

Educommunication and organizational communication: a universe of possibilities and connections

Educomunicación y comunicación organizacional: un universo de posibilidades y conexiones

\section{Larissa Bortoluzzi Rigo}

- Doutoranda em Comunicação Social na Pontifícia Universidade Católica do Rio Grande do Sul (PUC-RS)

- Mestre em Literatura Comparada pela Universidade Regional Integrada do Alto Uruguai e das Missões (URI) - Frederico Westphalen

- Graduada em Jornalismo pela Universidade Federal de Santa Maria (UFSM) - Frederico Westphalen

- E-mail: Lary_rigo@yahoo.com.br

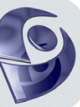

Ivana de Jesus Gehlen

- Mestranda em Comunicação Social na PUC-RS

- Graduada em Jornalismo pela PUC-RS

- E-mail: ivana.gehlen@acad.pucrs.br

Marcelo de Barros Tavares

- Mestrando em Comunicação Social na PUC-RS

- Docente do Centro Universitário Ritter dos Reis (UniRitter)

- E-mail: marcelotavares21@gmail.com

Keynayanna Késsia Costa Fortaleza

- Mestranda em Comunicação Social na PUC-RS

- Graduada em Jornalismo pela Faculdade Santo Agostinho (FSA) - Teresina, PI

- E-mail:keynayanna@hotmail.com 


\section{Resumo}

O artigo propõe uma reflexão a respeito das convergências entre educomunicação (Soares, 2009) e comunicação organizacional (Kunsch, 2016), pela perspectiva da cultura da informação (Jenkins, 2006; Shirky, 2011). 0 recorte teórico considera os ambientes organizacionais como espaços de interação, construção e disputa de sentidos (Baldisssera, 2008), num contexto marcado pela cultura participativa, em espaços de educação não-formal (Peruzzo, 2008). Por fim, a relação entre os conceitos envolvendo a educomunicação revelam novas possibilidades na área da comunicação nos ambientes organizacionais.

PALAVRAS-CHAVE: COMUNICAÇÃO • COMUNICAÇÃO ORGANIZACIONAL・CULTURA DA INFORMAÇÃO • EDUCOMUNICAÇÃO.

\section{Abstract}

The article proposes a reflection about the convergences between educommunication (Soares, 2009) and organizational communication (Kunsch, 2016), by the perspective of information culture (Jenkins, 2006; Shirky, 2011). The theoretical focus considers organizational environments as spaces of interaction, construction and dispute of senses (Baldisssera, 2008), in a context distinguished by participatory culture, in spaces of non-formal education (Peruzzo, 2008). Finally, the connection across the concepts related to educommunication reveals new possibilities in the area of communication in organizational environments.

\section{KEYWORDS: COMMUNICATION・ORGANIZATIONAL COMMUNICATION・INFORMATION CULTURE・EDUCOMMUNICATION.}

\section{Resumen}

El artículo propone una reflexión acerca de las convergencias entre educomunicación (Soares, 2009) y comunicación organizacional (Kunsch, 2016), por la perspectiva de la cultura de la información (Jenkins, 2006; Shirky, 2011). El recorte teórico considera los ambientes organizacionales como espacios de interacción, construcción y disputa de sentidos (Baldisssera, 2008), en un contexto marcado por la cultura participativa, en espacios de educación no formal (Peruzzo, 2008). Por último, la relación entre los conceptos que involucran la educomunicación revela nuevas posibilidades en el área de la comunicación en los ambientes organizacionales. 


\section{ANO 14 • NÚMERO 26 • 1ํSEM. 2017 • ORGANICOM \\ EDUCOMUNICAÇÃO E COMUNICAÇÃO ORGANIZACIONAL: UM UNIVERSO DE POSSIBILIDADES E CONEXÕES}

comunicação e a educação são dois campos de conhecimento distintos que oferecem subsídios para discussões sobre
suas incertezas e confrontos. As duas áreas sempre interagiram, mas, em especial nas últimas décadas, têm sido
objeto de pesquisa e debates por meio de um novo campo de interdisciplinaridade: a Educomunicação (Freire, 2001).

Diante de um tema que conta com restrita bibliografia no que diz respeito ao seu campo de atuação nas organizações, o objetivo desta reflexão é conectar pontos de ligação entre a educomunicação e a comunicação organizacional. Assumimos a noção de comunicação organizacional na perspectiva de Baldissera (2008), em que os ambientes organizacionais permeiam uma intensa disputa de sentidos entre os sujeitos envolvidos nas relações interpessoais e da organização com os seus mais diversos públicos.

Esse percurso é feito sob o prisma de um contexto de cultura da informação (Jenkins, 2008) vivenciado atualmente. Acreditamos ser relevante promover esta reflexão perpassando os conceitos e contextos que envolvem ambas as temáticas, a partir de um levantamento bibliográfico, ${ }^{1}$ para assim compreender, como esse recente campo de atuação e pesquisa relaciona educação, comunicação e organizações.

\section{INTERCONEXÕES ENTRE COMUNICAÇÃO E EDUCOMUNICAÇÃO}

Compreendemos que as relações que envolvem comunicação e educação nos remetem a um contexto sócio-histórico marcado pela linguagem e pelas formas de estabelecer relacionamentos através da exposição de diálogos. Ao longo dos anos, a sociedade passou a produzir maneiras inéditas de se relacionar e interagir, configurando um tipo de comunicação mais diversificado ou, pelo menos, mais analisado em suas nuances.

Remontando o contexto histórico do tema, Citelli (2009) menciona que no início do século XX, já havia influência televisiva sobre os estudos referentes às relações entre educação e comunicação. Surgiram projetos de ordem educacional envolvendo ações comunicativas. Na década de 1970, a leitura crítica a respeito desse tipo de meio de difusão expandiu-se "como caminho para a formação da consciência crítica das audiências" (Soares, 2014, p. 9). Nesse momento, a proliferação para outros meios e direções passou a se tornar foco de estudo para pesquisadores no mundo todo, que refletiam sobre a educação e a comunicação. Estudiosos como Baccega (2009), Citelli (2009), Freire (2001), Kaplún (1999), Soares (2009), dentre outros, foram determinantes para a nova área.

As interconexões entre comunicação e educação, portanto, datam de circunstâncias históricas, mas presentes de forma evidente na atualidade. As pesquisas e os debates sobre as novas maneiras de aprendizado fora dos ambientes propriamente ditos escolares, ou seja, a educação não-formal, ganham novos investimentos e possibilidades, já que essa modalidade propicia o diálogo e a formação de cidadãos mais críticos. Por isso, consideramos relevante compreender o fenômeno no intuito de expor novas possibilidades de ampliar o debate a respeito do assunto nas organizações objetivando contemplar 0 indivíduo nelas inserido, dentro do contexto de uma era de cultura da informação².

Após o surgimento da educomunicação, observamos no processo de redemocratização do país, na década de 1980, como inferem Kunsch (2003) e Nassar (2012), o desenvolvimento dos investimentos e das pesquisas referentes à comunicação

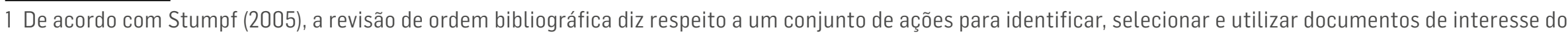
pesquisador para sua pesquisa.

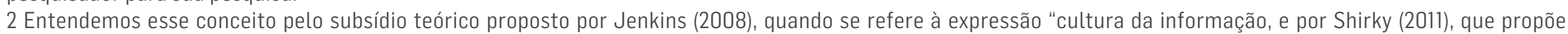

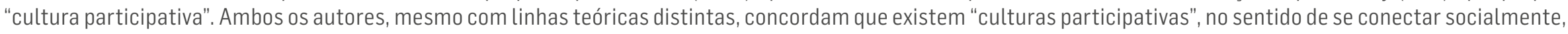
construindo uma rede que partilha conhecimentos e experiências.
} 


\section{ANO 14 • NÚMERO 26 • 1ํㅗㄴ. 2017 • ORGANICOM}

EDUCOMUNICAÇÃO E COMUNICAÇÃO ORGANIZACIONAL: UM UNIVERSO DE POSSIBILIDADES E CONEXÕES

organizacional no Brasil. Isso disseminou novas reflexões para um mercado em expansão, gerando desafios significativos para gestores e profissionais da área. 0 debate sobre conceitos e campos de atuação foi protagonizado, em grande parte, por Kunsch $(1986,2003,2009,2013,2016)$ e Torquato (1986), ambos de forma pioneira, além de outros que pontuam determinadas características da comunicação organizacional, como Baldissera (2008), Nassar (2012), Nassar e Cogo (2013) etc.

Consideramos que, ao tornar-se uma estratégia (Pérez; Massoni, 2009) ${ }^{3}$ nas organizações, a comunicação tem respaldo para se tornar um fator decisivo em ações, relacionamentos e projeções, atribuindo, assim, significados sociais e possibilidades de contemplação das dimensões humana e cultural dos indivíduos, conforme preconiza Kunsch (2016). A autora propõe redimensionar a visão da comunicação estratégica vigente, que é conservadora, ponderando que é necessário vê-la de forma mais holística, "capaz de interpretar hermeneuticamente o mundo contemporâneo" (Kunsch, 2016, p. 37). Nesse sentido, a pesquisadora sugere que se trabalhe nessa perspectiva, e faz emergir, então, possibilidades de correlação com as práticas educomunicativas.

Expomos, portanto, algumas possibilidades com base num recorte de autores das áreas da comunicação, da educação, da educomunicação e da comunicação organizacional para dar suporte a uma proposta de atendimento às dimensões que humanizam as organizações e democratizam a educação. Conceitualmente, consideramos possível identificar demandas práticas de educomunicação nos ambientes organizacionais, partindo da premissa de que esses são espaços de disputa de sentidos e estão alicerçados por um contexto da cultura da informação. Entendemos que a triangulação desses conceitos - educomunicação, comunicação organizacional, cultura da informação - revela uma delimitação importante para os estudos da área. Para tanto, é importante contextualizar o ambiente organizacional onde estão inseridos os indivíduos, para então desenvolver ideias acerca do surgimento do campo da educomunicação e da cultura da informação, abrangendo as dimensões humanísticas nesse ambiente.

\section{O AMBIENTE ORGANIZACIONAL}

Não são raros os casos em que a dimensão humana do indivíduo em uma organização fica esquecida. É possível que seja próprio do comportamento organizacional ter um discurso que contemple essa dimensão, mas, às vezes, no intuito de priorizar o esforço de tornar o indivíduo produtivo e eficaz, atuam de outra forma no cotidiano. A dimensão humana, quando ganha esse sentido de produção de resultados, perde força, ou quase inexiste. É nessa linha de pensamento que Chanlat (1993, p. 23) faz constatações considerando que o campo organizacional é heterogêneo:

Herdeiro simultaneamente das relações humanas, de alguns ramos da sociologia industrial, da sociologia e das teorias das organizações, da abordagem sociotécnica, da dinâmica de grupo, do behaviorismo anglo-saxão, das diferentes correntes do management, o comportamento organizacional apresenta-se hoje como uma imensa colcha de retalhos, um campo aberto a quase todos os ventos teóricos.

0 autor, de forma pontual, apresenta algumas dimensões humanas que considera ausentes nos ambientes organizacionais: a cognitiva e da linguagem; a espaço-temporal; a psíquica e afetiva; a simbólica; a da alteridade; e a psicopatológica. A avaliação é de que o campo organizacional desenvolveu um comportamento isolado em que se negligenciam os conhecimentos atuais das ciências humanas básicas e de que este isolamento da produção intelectual tem duas causas. A primeira considera a hegemonia econômica, quantitativa que permeia as organizações e a sociedade, parecendo ter esquecido o resto, isto é, tudo o que não é redutível à formalização (Gorz, 1989). A segunda, "como todo o recurso a um pensamento externo e

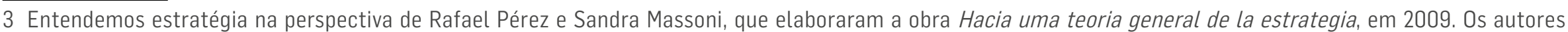

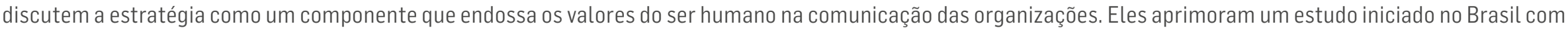
a pesquisadora Margarida Kunsch, que estudou o conceito dentro da comunicação organizacional. 
forçosamente crítico pode sempre ameaçar a ordem organizacional estabelecida, o mundo da gestão tem com frequência preferido as visões que são menos incômodas" (Chanlat, 1993, p.23). Nessa leitura, as organizações se veem inseridas em um contexto de valoração do âmbito material, as pessoas dentro das organizações ganham uma dimensão aproximada e a relação entre sujeitos somente toma uma dimensão humana quando passa por uma condição extraordinária. Do contrário, essas relações estão subordinadas a algum ganho, uma troca referente a um bem individual. Normalmente as causas se conectam com um pensamento voltado às mercadorias, para uma racionalidade econômica vigente. Chanlat (1993) evidencia esse pensamento quando se refere a uma percepção que vislumbra numerosos trabalhadores, principalmente jovens, numa tendência de procurar cada vez mais a realização pessoal fora do seu trabalho tradicional, além de diversos "executivos e golden boys das finanças", que "não parecem mais manter nenhuma lealdade com relação às instituições para as quais trabalham" (Chanlat, 1993, p. 25). Essa afirmativa remete-nos a ponderar que os funcionários das organizações podem estar buscando mais liberdade, mais independência, mas também mais autonomia para protagonizar ações efetivas de transformação da sociedade e de si mesmos, por meio da organização em que estão inseridos.

A antropologia das organizações diz respeito justamente ao objetivo de colocar em evidência dimensões ignoradas no âmbito dos conjuntos organizacionais, pois isso reafirma o papel do indivíduo, do simbólico, mas, ao mesmo tempo, reitera o seu lugar social. Enquanto ator do seu próprio espaço, que acreditamos estar presente na iniciativa da proposta de educomunicação nas organizações, o indivíduo inserido no meio organizacional se conecta com o protagonismo que lhe é devido e que lhe é assegurado quando a gestão amplia sua visão dialética para com e na sociedade. É nesse sentido que a cultura intervém inferindo uma dimensão própria das relações, que insere o indivíduo no domínio das experiências e do cotidiano. Para França (2013), a cultura não é um estoque inerte ou patrimônio de ideias, mas impulsiona indivíduos. Diante de uma matéria viva e atuante, estes são capazes de se revestir do seu próprio modo cultural, mesmo nas organizações em que estão inseridos, para se tornarem agentes de participação mútua entre aqueles que com eles convivem. Inserem-se atuantes na coletividade.

Comunicação e cultura estão inscritas em uma dinâmica reflexiva que atua sobre outra dinâmica reflexiva (aquela do processo interacional); a cultura é o contexto institucional de valores, papéis e posições que alimenta e fornece os parâmetros para a realização de determinada prática comunicativa. Esta, no entanto, marcada pela intervenção viva dos sujeitos, por uma sequência de estímulos, respostas, escolhas e posicionamentos - instruídos, mas imprevisíveis - é tanto o lugar de atualização e reprodução da cultura, como o lugar de sua renovação (França, 2013, p. 97).

Por essa visão, podemos apreender que se comunicar dentro do ambiente de extradição da cultura não é apenas transmitir um conteúdo, mas ali mesmo produzir, reproduzir, recriar. Algumas situações que permeiam esses caminhos são viabilizadas pelos processos de comunicação formais. Segundo a autora, as formas de alcance desse objetivo estão no âmago das organizações, por exemplo, ao criarem públicos onde havia indivíduos isolados e estabelecendo o diálogo num ambiente em que o silêncio impera (França, 2013). E tudo isso poderia ser feito a partir do indivíduo e não sobre ele.

Destacamos ainda a importância do entendimento de que as situações não são totalmente criadas em nossas mentes, mas são orientadas pelo cultural: "A cultura da organização, o sistema das significações da sociedade em que vivemos. É importante também nos darmos conta de que tais práticas são ativadoras - eventualmente modificadoras - da cultura da organização" (França, 2013, p. 97). A remodelagem dos ambientes organizacionais é promulgadora de indivíduos capazes de dialogar e de estabelecer pontos de conexão com o modelo coletivo que os cerca e que, de modo contínuo, modifica a cultura de uma sociedade. Pois indivíduos capazes de dar voz à própria voz têm mais chance, e mais coragem, de recriar ambientes que os façam mais felizes. Essa proposta pode ser possibilitada por uma área da educação que se alia à comunicação e projeta o indivíduo no sentido da almejada autonomia sem perdas, ou seja, a educomunicação. 


\section{EDUCOMUNICAÇÃO: UM NOVO CAMPO DE CONVERGÊNCIA}

A história da educomunicação está diretamente ligada às interfaces provocadas pela prática da educação junto com a Comunicação (Braga; Calazans, 2001). Kaplún (1999) foi um dos primeiros pesquisadores na América Latina ao defender, ainda na década de 1970, a comunicação não apenas como uma vertente midiática, mas, ultrapassando a visão redutora, ele considerava a área como um componente pedagógico. Para Freire (2001), ao tratarmos de educação, estamos nos atendo à comunicação. A união dessas temáticas foi pauta central de dois congressos da Intercom ${ }^{4}$, que com um intervalo de trinta anos, abordou a temática em diferentes perspectivas.

A discussão a respeito do novo campo de estudos no Brasil precede as reflexões propostas por Postman (1984), sobre a preparação das crianças para o mercado de trabalho, e, posteriormente, por Buckingham (2000) ao pesquisar as possibilidades das mídias nos ambientes escolares. Com diferentes perspectivas, o primeiro autor analisou, com um tom crítico, o impacto das mídias no universo infantil, sobretudo após o surgimento da televisão na sociedade estadunidense. $\mathrm{E}$ o segundo estudo, elaborado pelo pesquisador britânico, apresenta o viés positivo da utilização dos meios de comunicação no processo de ensino-aprendizagem, desenvolvido nas escolas.

Partindo desses diálogos preliminares sobre a educação midiática, tensionada pelas possibilidades propiciadas pela comunicação dentro dos espaços educativos, surgiram correntes na América Latina, conduzidas principalmente por Freire (2001), Kaplún (1999), Citelli (2009) e Soares (2009). Nesse contexto, a educomunicação emerge como uma prática social que promove a humanização nas relações, constituindo:

um paradigma gerador das ações voltadas a criar e desenvolver "ecossistemas comunicativos" abertos e democráticos nos espaços educativos, mediados pelas tecnologias da comunicação, tendo como meta construir e favorecer processos educativos que se voltam ao pleno exercício do direito da expressão, a serviço da prática da cidadania (Soares, 1999, p. 50).

Essa visão latino-americana surge da perspectiva dialógica e participativa da comunicação, convergindo os olhares em torno da educação, que deixa de ser apenas um mercado para os comunicadores, mas revela-se como um campo de conhecimento determinante para os avanços na valorização das dimensões humanas e também na compreensão dos efeitos possíveis da cultura da informação. As experiências adquiridas no Núcleo de Comunicação e Educação da Universidade de São Paulo (NCE-USP), por exemplo, trouxeram uma importante contribuição para o desenvolvimento acadêmico, ao desvendar a prática da comunicação em comunidades escolares da cidade de São Paulo. Entretanto, os relatos retomam as discussões sobre a comunicação popular (Peruzzo, 1999), a era da participação (Bordenave, 1992) e a comunicação participativa (Kaplún, 1984). Esse cenário está relacionado à democratização da sociedade, especialmente pelo viés da realidade brasileira, em que os espaços educativos sofrem as influências dos movimentos sociais, e a comunicação passa a ser considerada como "meio de conscientização, mobilização, educação política, informação e manifestação cultural do povo" (Peruzzo, 1999, p. 125). Ainda nessa perspectiva,

a comunicação popular se desenvolve então relacionada às necessidades de expressão e organização desse movimento de negação e, ao mesmo tempo, de construção de uma sociedade nova. Ela está articulada a um processo de consciência-organização-ação mais amplo de setores de classes subalternas (Peruzzo, 1999, p. 36).

\footnotetext{
4 A Sociedade Brasileira de Estudos Interdisciplinares da Comunicação foi fundada em 1977 para fomentar a troca de conhecimento entre pesquisadores e profissionais, em todas as áreas do campo da comunicação. Anualmente, ela realiza um congresso nacional, precedido de cinco congressos regionais. 0 evento nacional é considerado um dos maiores da comunicação.
} 
Com vistas para essa realidade, a Organização das Nações Unidas (ONU) se envolveu e incentivou as pesquisas que surgiram na América Latina desde a década de 1960. Hoje o Brasil está entre as referências na área e produz grande número de pesquisas e discussões junto a outras nações. Os pesquisadores da Universidade de São Paulo (USP) atuam na defesa da educomunicação, que, de acordo com Soares (2009, p. 161-162), diz respeito ao

conjunto das ações inerentes ao planejamento, implementação e avaliação de processos comunicativos, assim como de programas e produtos com intencionalidade educativa, destinados a criar e fortalecer ecossistemas comunicativos abertos, criativos, sob a perspectiva da gestão compartilhada e democrática dos recursos da informação.

Com base no seu conceito e diante de suas possibilidades de atuação nas organizações, compreendemos que a educomunicação se destaca promovendo ações e formas de planejamento que remetem a processos de ordem comunicacional e educativa através da difusão do saber, conhecimento e apropriação de diálogos. De acordo com Citelli (2011), a educomunicação sinaliza novos mecanismos de produção, circulação e recepção de conhecimento e da própria informação. Desse modo, essa área de atuação, revela um conceito, que ao ser praticado, pode beneficiar os ambientes empresariais, principalmente num tempo marcado pela hegemonia da comunicação e da cultura da informação. Estas perspectivas são alicerçadas pelo viés do "ecossistema comunicativo", exposto por Martín-Barbero (2014), ao discutir as convergências da comunicação e da educação e pontuar que as duas áreas têm fator preponderante para a formação dos sujeitos de um determinado grupo social.

\section{PRÁTICAS NUM CONTEXTO DE CULTURA DA INFORMAÇÃO}

A reflexão sobre o entendimento da educomunicação, enquanto um paradigma (no sentido de orientação) para os atores sociais, permite aludir às convergências entre esse campo e o contexto atual atribuído à comunicação organizacional, levando em consideração as dimensões propostas por Kunsch (2016). Essas conexões centram-se em algumas observações: se a educomunicação se relaciona pelo paradigma/orientação aos atores sociais, a posição que esses sujeitos ocupam dentro desse ambiente deve ser considerada. Nesse sentido, a obra The centrality of culture: notes on the cultural revolutions of our time, de Stuart Hall (1997), auxilia na compreensão de identificar as noções que envolvem a centralidade da cultura e o seu papel na constituição dessa teia que é a vida em sociedade.

A pergunta que permeia as reflexões de Hall (1997, s.p.) é: "Por que a cultura se encontra no centro de tantas discussões e debates, no presente momento?". A reflexão enfatiza a importância que a cultura tem frente às ciências sociais e humanas, já que "os seres humanos são seres interpretativos, instituidores de sentido" (Hall, 1997, s.p.). Assim, por meio dos sistemas de significados, é que ocorre a organização de conduta social. 0 autor intitula como cultura "toda ação social [que] é 'cultural'; todas as práticas sociais expressam ou comunicam um significado e, nesse sentido, são práticas de significação" (Hall, 1997, s.p.). Por esse propósito, imbuídos de "práticas de significação", os atores sociais constituem suas identidades e subjetividades.

0 conceito de identidade é compreendido por Hall (2006) em justaposição à teoria social. Seu principal argumento reside na(s) velha(s) identidade(s), ou, atrelando ao nosso objeto de estudo, a imersão de uma nova identidade, a passagem de um sujeito unificado para um sujeito fragmentado, resultando em uma "crise de identidade" (Hall, 2006, p. 7). A ideia apontada pelo autor é "parte de um processo mais amplo de mudança, que está deslocando as estruturas e processos centrais das sociedades modernas e abalando os quadros de referência que davam para os indivíduos uma ancoragem estável no mundo social" (Hall, 2006, p. 7). A partir desses preceitos, Hall entende o conceito de identidade no plural, identidade(s), já que os sujeitos as transformam de acordo com o contexto social em que estão inseridos. 


\section{ANO 14 • NÚMERO 26 • 1ํSEM. 2017 • ORGANICOM \\ EDUCOMUNICAÇÃO E COMUNICAÇÃO ORGANIZACIONAL: UM UNIVERSO DE POSSIBILIDADES E CONEXÕES}

O segundo ponto de intersecção da relação entre a educomunicação e a comunicação organizacional se relaciona ao contexto dos atores sociais frente às identidades (Hall, 2006). Estas se direcionam para determinadas práticas alusivas à cultura da informação, ou ao que elucidam Dugay (1994) e Jenkins (2008): "interconexões globais". Nesse cenário, as formas de comunicação convergem para a "cultura da informação" (Jenkins, 2008) ou, ainda, a "cultura participativa" (Shirky, 2011). Os autores, mesmo com proposições distintas, concordam com o entendimento de que existem "culturas participativas". No âmbito de compartilhar, os participantes se conectam socialmente, construindo uma rede que partilha conhecimentos e experiências.

Nessa aproximação social de (com)partilhar, ou seja, tornar comum, há um movimento de engajamento cívico: "Nem todos os membros precisam contribuir, mas todos devem acreditar que são livres para contribuir quando estiverem prontos e que suas contribuições serão devidamente valorizadas" (Jenkins, 2006, s.p., tradução livre). Para ilustrar a delimitação dessa liberdade, resgatamos aqui o entendimento de Peruzzo (2009) sobre comunicação popular. Esta também é denominada de "alternativa, participativa, participatória, horizontal, comunitária, dialógica e radical, dependendo do lugar social, do tipo de prática em questão e da percepção dos estudiosos" (Peruzzo, 2009, p. 47). No entanto, em seu sentido político relaciona-se às formas de expressão de alguns segmentos mais pobres da população, ainda que estes estejam em processo de mobilização no intuito de suprir necessidades e fazer vigorar sua participação política em direção à justiça social. "No entanto, desde o final do século passado passou-se a empregar mais sistematicamente, no Brasil, a expressão comunicação comunitária para designar este mesmo tipo de comunicação, ou seja, seu sentido menos politizado" (Peruzzo, 2009, p. 47). A pesquisadora denota que o conceito de "comunicação comunitária" se inspira em Paulo Freire, sobretudo, na dialogicidade da educação e seu papel de orientação, no que tange à transformação do ser humano no mundo.

Junto com as prerrogativas dos conceitos atribuídos por Peruzzo (2009), é preciso considerar as pesquisas de Citelli (2009). 0 estudioso apresenta outras alternativas pertinentes à educação, tais como as denominadas fora dos ambientes escolares, a educação não-formal, o que nos direciona para novos olhares na relação entre a comunicação e a educação, ou como a primeira pode ser aliada da segunda diante de seus públicos.

A junção entre o papel da cultura participativa (no sentido de se conectar socialmente), estritamente relacionada à comunicação comunitária, transporta ao pensamento de que a informação via tecnologias e educação forma uma base para constituir as interfaces presentes nos preceitos relativos à educomunicação, inclusive dentro das organizações. Aliando os movimentos da comunicação popular e as possibilidades de educação não-formal, em que existem processos sistêmicos, mas sem estrutura curricular, apreendemos uma interessante esfera para o estudo da influência da cultura da informação nos ambientes organizacionais a partir das práticas de educomunicação.

\section{CONSIDERAÇÕES FINAIS}

Numa era de expansão de processos comunicativos, a educomunicação apresenta-se como possibilidade. Elucidar relações e interfaces sobre as possibilidades de atuação nas áreas da comunicação e educação nos faz ponderar as várias potencialidades que a educomunicação sinaliza. Sobre a sua atuação nas organizações, compreendemos que a mesma está conectada à disseminação do saber, à promoção do diálogo, à gestão do conhecimento, aos incentivos ligados à questão da humanização no ambiente de trabalho, demonstrando, assim, uma proposta voltada a formar cidadãos mais críticos, com maior poder de decisão. 
Isso tudo nos coloca diante de um contexto que é marcado pela "cultura participativa" (Shirky, 2011), isto é, as vivências em uma rede que partilha conhecimentos e experiências. É diante dessa visão que Baccega (2009) revela que é neste campo que se constroem sentidos novos, a organização se coloca como produtora de significados sociais, pois a mesma também desenvolve um papel regulador na sociedade. Com este recorte teórico, apoiados pelo viés da comunicação organizacional de Baldissera (2008), temos a possibilidade de inferir que as organizações se revelam como espaços de construção de sentidos e significados em que as práticas educomunicativas podem fomentar espaços democráticos que incentivam a prática da cidadania.

Portanto, refletimos que difundir o saber em escala empresarial, mesmo com todos os desafios que essas práticas inferem em um contexto social de valoração dos bens em detrimento da dimensão humana dos indivíduos, pode trazer benefícios e novas oportunidades em um nível organizacional e humano. Acreditamos, desta maneira, que as práticas educomunicativas não se limitam aos ambientes escolares, mas que o ecossistema comunicativo (Martín-Barbero, 2014) privilegia espaços abertos e democráticos nos contextos organizacionais, e, assim como suas ações e produtos, a organização é constituída como um ambiente de construção de significados. Nesse sentido, retomamos Kunsch (2016), que fala na coerência no discurso organizacional e atribui o significado disso à qualificação das dimensões, principalmente, humana e cultural, que defendemos neste estudo como a base para o entendimento da relevância de difundir uma cultura educomunicativa nos ambientes organizacionais.

A apropriação das práticas educomunicativas, no contexto das organizações, pode revelar um objeto fértil de pesquisa para o campo científico da comunicação organizacional ao considerar as formas de relacionamento e as formas de produção comunicativa nesses ambientes. Acreditamos que elas podem ampliar a possibilidade de interação do sujeito, influenciado pela cultura da informação, para que se aproprie dos saberes que permeiam seu conjunto cultural. A partir do viés antropológico, esses ambientes constituem-se como cenários da interação social, de troca e de compartilhamento de culturas e construção de sentidos.

A educomunicação, enquanto um campo de convergência e de interfaces, possibilita potencialidades nos estudos das práticas sociais, principalmente quando se trata dos ecossistemas comunicativos. Sendo assim, ela se apresenta como um conceito a ser colocado em debate pelos efeitos que pode inferir na busca da valoração do sujeito e das práticas em favor das dimensões humanas e culturais. Nesta reflexão, ao ser relacionada à comunicação organizacional e ao conceito de cultura da informação, a educomunicação revela-se como uma potencialidade de pesquisa dentro da comunicação nos ambientes organizacionais, ao constituir-se como uma prática social que fomenta o exercício do direito de expressão e a atuação efetiva da cidadania.

\section{REFERÊNCIAS}

BALDISSERA, Rudimar. Comunicação organizacional: uma reflexão possível a partir do paradigma da complexidade. In: OLIVEIRA, Ivone de L.; SOARES, Ana Thereza N. (Orgs.). Interfaces e tendências da comunicação no contexto das organizações. São Caetano do Sul, SP: Difusão, 2008. p. 149-77.

BACCEGA, Maria Aparecida. Campo comunicação/educação: mediador do processo de recepção. In: BACCEGA, Maria Aparecida; COSTA, Maria Cristina Castilho (Orgs.). Gestão da comunicação: epistemologia e pesquisa teórica. São Paulo: Paulinas. 2009. p. 13-26. 
BORDENAVE, Juan E. Diaz. O que é participação. São Paulo: Brasiliense, 1992.

BRAGA, José Luiz; CALAZANS, Regina. Comunicação e educação: questões delicadas na interface. São Paulo: Hacker Editores, 2001.

BUCKINGHAM, David. After the death of childhood: growing up in the age of the electronic media. Cambridge: Polity, 2000.

CHANLAT, Jean-François. O indivíduo na organização: dimensões esquecidas. Trad. de Ofélia de Lanna Tôrres. 2. ed. São Paulo: Atlas, 1993.

CITELLI, Adílson. Comunicação/ Educação: situações. In: BACCEGA, Maria Aparecida; COSTA, Maria Cristina Castilho (Orgs.). Gestão da comunicação: epistemologia e pesquisa teórica. São Paulo: Paulinas, 2009.

. Comunicação e educação: implicações contemporâneas. In: CITELLI, Adilson Odair; COSTA, Maria Cristina Castilho (Orgs). Educomunicação: construindo uma nova área de conhecimento. São Paulo: Paulinas, 2011. p. 59-76.

DUGAY, Paul. Making up managers: bureaucracy, entreprise and the liberal art of separation. British Journal of Sociology, v. 45, n. 4, p. 655-674, 1994.

FRANÇA, Vera. V. Comunicação e cultura: relações reflexivas em segundo grau. In: MARCHIORI, Marlene (Org.). Comunicação em interface com a cultura. São Caetano do Sul, SP: Difusão, 2013.

FREIRE, Paulo. Extensão ou comunicação?Rio de Janeiro: Paz e Vida.2001.

GORZ, André. Crítica da divisão do trabalho. 2. ed. São Paulo: Martins Fontes, 1989.

HALL, Stuart. The centrality of culture: notes on the cultural revolutions of our time. In: THOMPSON, Kenneth (Org.). Media and cultural regulation. London: Sage. Tradução brasileira: A centralidade da cultural: notas sobre as revoluções culturais do nosso tempo. Revista Educação e Realidade, Porto Alegre, v. 22, n. 2, p. 15-46, jul./dez.1997.

A identidade cultural na pós-modernidade. Trad. de Tomaz Tadeu da Silva e Guaciara Lopes Louro. 11. ed. Rio de Janeiro: DP \& A Editora, 2006. [Título original: The question of cultural identity].

JENKINS, Henry. Confronting the challenges of participatory culture. 2006. Disponível em: <http://henryjenkins.org/2006/10/ confronting_the_challenges_of.html\#sthash. Z3zuFS3V.dpuf>. Acesso em: 25 fev. 2017.

Convergence culture: la cultura de la convergencia de los medios de comunicação. Barcelona: Paidós, 2008.

KAPLÚN, Mario. Comunicación entre grupos: el método del cassette-foro. Bogotá, Colômbia, Centro Internacional de Investigaciones para el Desarrollo, 1984.

Processos educativos e canais de comunicação. Comunicação \& Educação, v. 5, n. 14, p. 68-75, jan/abr.1999. Disponível em: Disponível em: <http://www.revistas.usp.br/comueduc/article/view/36846>. Acesso em: 24 jan. 2017.

KUNSCH, Margarida M. Krohling. Comunicação organizacional. Vol. 1. Histórico, fundamentos e processos. São Paulo: Saraiva. 2009.

Planejamento de relações públicas na comunicação integrada. [1986]. 4. ed. - rev., atualiz. e ampl. São Paulo: Summus, 2003 
Comunicação organizacional: conceitos e dimensões dos estudos e das práticas. In: MARCHIORI, Marlene (Org.). Comunicação em interface com cultura. Rio de Janeiro: Senac. 2013.

. A comunicação nas organizações: dos fluxos lineares às dimensões humana e estratégica. In: KUNSCH, Margarida M. Krohling. (Org.). Comunicação organizacional estratégica: aportes conceituais e aplicados. São Paulo: Summus, 2016. p. 37-58.

MARTín-BARBERO, Jesús. A comunicação na educação. Trad. de Maria Immacolata Vassallo de Lopes; Dafne Melo. São Paulo: Contexto, 2014.

NASSAR, Paulo; COGO, Rodrigo Silveira. Memória e narrativa organizacional como expressões da cultura organizacional: 0 poder do storytelling. In: MARCHIORI, Marlene (Org.). História e memória. São Caetano do Sul, SP: Difusão, 2013.

NASSAR, Paulo. Relações públicas: a construção da responsabilidade histórica e o resgate da memória institucional das organizações. 3. ed. São Caetano do Sul, SP: Difusão, 2012.

PÉREZ, Rafael; MASSONI Sandra. Hacia uma teoria general de la estratégia. Barcelona: Ariel Editores, 2009.

PERUZZO, Cicilia M. Krohling. Comunicação nos movimentos populares. a participação na construção da cidadania. 2. ed. Petrópolis, RJ: Vozes, 1999.

. O lugar da comunicação comunitária nas políticas públicas do Brasil. In: ENCONTRO DA COMPÓS, XVII, Associação Nacional dos Programas de Pós-Graduação em Comunicação - GT de Economia Política e Políticas de Comunicação, Unip, São Paulo, junho de 2008. Disponível em: <http://www.compos.org.br>. Acesso em: 24 fev. 2017.

Conceitos de comunicação popular, alternativa e comunitária revisitados e as reelaborações no setor. ECO-Pós, v.12, n.2, p. 46-61, maio/ago. 2009.

POSTMAN, Neil. The disappearance of childhood. London: Penguin, 1984.

SHIRKY, Clay. A cultura da participação: criatividade e generosidade no mundo conectado. Trad. de Celina Portocarrero. Rio de Janeiro: Zahar, 2011.

SOARES, Ismar de Oliveira. Comunicação/educação: a emergência de um novo campo e o perfil de seus profissionais. Contato: Revista Brasileira de Comunicação, Arte e Educação, Brasília, DF, a. 1, n. 2, p. 19-74, jan/mar. 1999.

- Caminhos da gestão comunicativa como prática de educomunicação. In: BACCEGA, Maria Aparecida; COSTA Maria Cristina Castilho (Orgs.). Gestão da comunicação; epistemologia e pesquisa teórica. São Paulo: Paulinas, 2009. p. 161-188.

. Introdução à edição brasileira. In: APARICI, Roberto (Org.). Educomunicação para além do 2.0. São Paulo: Paulinas, 2014.

STUMPF, Ida Regina C. Pesquisa bibliográfica. In: DUARTE, Jorge; BARROS, Antônio (Orgs). Métodos e técnicas de pesquisa em comunicação. São Paulo: Atlas, 2005.

TORQUATO, Francisco Gaudêncio. Comunicação empresarial/Comunicação institucional: conceitos, estratégias, sistemas, estrutura, planejamento e técnicas. São Paulo: Summus, 1986. 